Z Gerontol Geriat 2022 $\cdot 55: 151-156$ https://doi.org/10.1007/s00391-022-02016-8 Eingegangen: 12. Juni 2021 Angenommen: 17. Dezember 2021 Online publiziert: 21. Januar 2022

(C) Der/die Autor(en) 2022

\section{Strategien, Richtlinien und Empfehlungen zur Bewältigung der COVID-19-Pandemie in Einrichtungen der Palliativ- und Hospizversorgung. Ergebnisse eines Scoping-Reviews}

\author{
Diana Wahidie' · Kübra Altinok' · Yüce Yılmaz-Aslann ${ }^{1,2,3}$ • Patrick Brzoska' \\ ${ }^{1}$ Fakultät für Gesundheit/Department für Humanmedizin, Lehrstuhl für Versorgungsforschung, \\ Universität Witten/Herdecke, Witten, Deutschland; ${ }^{2}$ Fakultät für Gesundheitswissenschaften, AG3 \\ Epidemiologie \& International Public Health, Universität Bielefeld, Bielefeld, Deutschland; ${ }^{3}$ Fakultät für \\ Gesundheitswissenschaften, AG6 Versorgungsforschung und Pflegewissenschaft, Universität Bielefeld, \\ Bielefeld, Deutschland
}

Zusammenfassung

Hintergrund: Palliativ- und Hospizeinrichtungen sind durch die COVID-19-Pandemie besonderen Herausforderungen ausgesetzt. Gründe dafür sind insbesondere das hohe Alter und bestehende Vorerkrankungen der Patienten sowie ein körperlich naher Kontakt zwischen Mitarbeitern und Patienten.

Ziel der Arbeit: Ziel der vorliegenden Studie ist es, bestehende Strategien, Richtlinien und Empfehlungen zum Umgang mit den Herausforderungen der COVID-19-Pandemie in der Palliativ- und Hospizversorgung zu ermitteln, die in Deutschland und anderen Ländern zum Einsatz kommen.

Material und Methoden: Im Rahmen eines Scoping Reviews wurden die Datenbanken PubMed, CINAHL, Web of Science und Psyclnfo durchsucht. Zusätzlich wurde "graue Literatur" über Google Search und Google Scholar recherchiert. Eingeschlossen wurden alle deutsch- und englischsprachigen Artikel im Zeitraum Januar 2020 bis August 2021, die sich auf die stationäre Palliativ- und Hospizversorgung beziehen. Ergebnisse: Insgesamt wurden 51 Veröffentlichungen in die Analyse aufgenommen. Die identifizierten Maßnahmen lassen sich 10 verschiedenen Kategorien zuordnen: Maßnahmen zur Infektionskontrolle, strukturelle Maßnahmen, Besuchsregelungen, Kommunikationsstrukturen, Schulung und Aufklärung, psychosoziale Unterstützungsmaßnahmen, spezifische Überlegungen für Menschen mit Demenz, vorausschauende Pflegeplanung, Abschiednahme sowie Maßnahmen nach dem Tod.

Diskussion: Bei der Bewältigung der COVID-19-Pandemie dominieren insbesondere Maßnahmen zur Ermöglichung der Kommunikation zwischen Patienten, Mitarbeitern und Angehörigen, psychosoziale Unterstützungsmaßnahmen, strukturelle Maßnahmen und Empfehlungen zur Schulung und Aufklärung aller Beteiligten. Strategien zur Palliativversorgung von Menschen mit Demenz in Pandemiezeiten sowie zu Abschiednahme und Trauerbewältigung von Hinterbliebenen finden weniger Beachtung.

Schlüsselwörter

SARS-CoV-2 $\cdot$ Maßnahmen · Social Distancing $\cdot$ Infektionskontrolle $\cdot$ Public-Health-Krise 


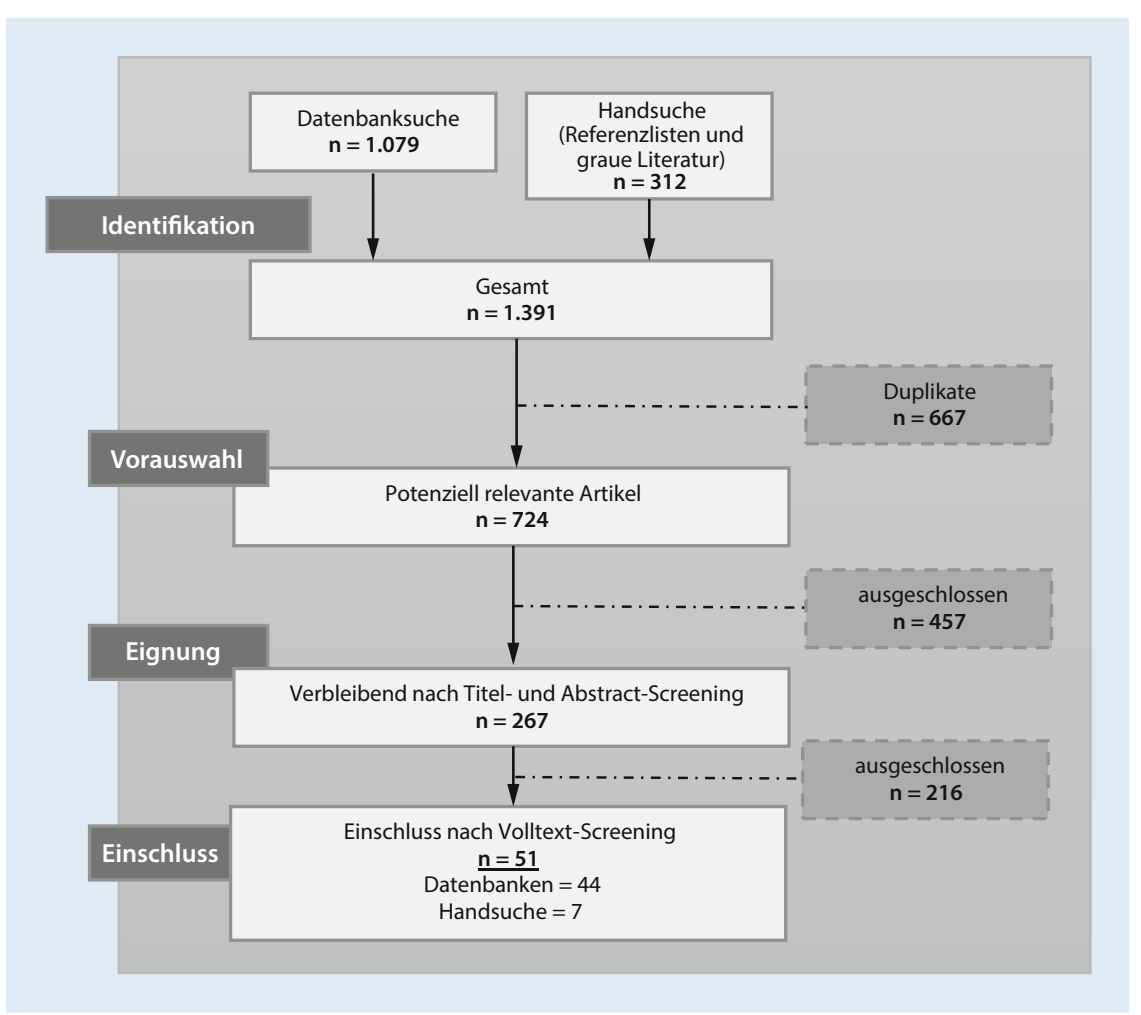

Abb. 1 \ Flussdiagramm zur Darstellung des Prozesses der Publikationsauswahl

\section{Einleitung}

Durch die rasche Ausbreitung des SARSCoV-2-Virus (Severe acute respiratory syndrome coronavirus type 2) waren und sind v. a. Einrichtungen der Palliativ- und Hospizversorgung herausgefordert [1]. Patienten solcher Versorgungseinrichtungen sind aufgrund ihres häufig höheren Alters und bestehender Vorerkrankungen einem erhöhten Risiko für einen schweren Krankheitsverlauf von COVID-19 (Coronavirus Disease 2019) ausgesetzt [2]. Infolge des engen physischen Kontakts zwischen Mitarbeitern und Patienten bei der Durchführung pflegerischer Leistungen und der Vielzahl an Gemeinschaftsaktivitäten besteht zudem eine hohe Infektionsgefahr [3]. Die zur Senkung des Ansteckungsrisikos eingeführten Infektionsschutzmaßnahmen, wie z. B. Besuchs- und Ausgangsbeschränkungen, erhöhen aufgrund des Wegfalls von Unterstützungspersonen (z.B. Ehrenamtliche) die Arbeitsbelastungen des Personals und tragen gleichzeitig zu einer erschwerten Abschiednahme von sterbenden Patienten bei [4].

Um den aufgezeigten Herausforderungen zu begegnen, müssen Palliativ- und
Hospizeinrichtungen innovative Ansätze entwickeln, die den Bedürfnissen aller Akteure Rechnung tragen. Ziel des vorliegenden Beitrags ist es, bestehende Strategien, Richtlinien und Empfehlungen zum Umgang mit der COVID-19-Pandemie in der stationären Palliativ- und Hospizversorgung auf Basis verfügbarer internationaler Literatur zu ermitteln. Die Erkenntnisse des Beitrags können sowohl Leitungskräfte stationärer Einrichtungen als auch politische Akteure dabei unterstützen, ihre bisherigen Strategien und Maßnahmen im Umgang mit der COVID-19-Pandemie zu bewerten und ggf. im Hinblick auf zukünftige Public-Health-Krisen anzupassen.

\section{Methodik}

Zur Erfassung bestehender Strategien, Richtlinien und Empfehlungen wurde ein Scoping-Review aller in deutscher und englischer Sprache verfügbaren Publikationen aus dem Zeitraum 01.01.2020 bis 17.08.2021 in den Datenbanken PubMed, CINAHL, Psyclnfo und Web of Science sowie den Internetsuchmaschinen Google Search und Google Scholar durchgeführt. Anders als bei einem systematischen Re- view liegt der Fokus dabei nicht darauf, die Wirksamkeit bestehender Maßnahmen zu beurteilen - da bisher in der Regel kaum analytische Studien zum Thema vorliegen, wäre dies kaum möglich sondern darauf, zusammenfassend einen Überblick über einen neu entstandenen Forschungsbereich zu ermöglichen. Die Strukturierung erfolgte auf Basis eines fünfstufigen Vorgehens (Zusatzmaterial online) [5-7] und wird entlang der Empfehlungen der "Preferred Reporting Items for Systematic Reviews and Meta-Analyses - Extension for Scoping Reviews" (PRISMA-ScR) berichtet [8].

\section{Ergebnisse}

Aus insgesamt 1391 Treffern wurden 51 Artikel final in die Analyse eingeschlossen (- Abb. 1). Die identifizierten Strategien, Richtlinien und Empfehlungen lassen sich 10 verschiedenen Ebenen zuordnen, die im Folgenden erläutert werden (für nähere Informationen: Zusatzmaterial online, Tab. 3 und 4).

\section{Maßnahmen zur Infektionskontrolle}

Ein Großteil der Veröffentlichungen $(n=20)$ berichtet von der Einführung grundlegender Maßnahmen zur Infektionskontrolle. Dazu gehören die regelmäßige Reinigung der Stationen, Sprechzimmer und aller Oberflächen $[9,10]$, die Einführung von zusätzlichen Handwaschvorrichtungen $[11,12]$ und die strikte Einhaltung von Kontaktbeschränkungen [13, 14]. Darüber hinaus empfehlen die einbezogenen Artikel die regelmäßige Fensterlüftung [15], die Reduktion von Teambesprechungen, die Aussetzung weiterer "face-toface meetings", wie beispielsweise Supervisionen und Fortbildungen [16], und die Vermeidung der Versammlung von Personengruppen in engen Räumen [17]. Zudem wird empfohlen, dass nicht das gleiche Personal Patienten mit und ohne COVID-19 behandelt [18]. Weitere Maßnahmen zur Infektionskontrolle stellen das tägliche Gesundheitsscreening von Mitarbeitern und Patienten [19], die regelmäßige Testung der Mitarbeiter, Patienten, Angehörigen und anderer Besucher auf SARS-CoV-2 [10, 18, 20-22] sowie die 
Ausstattung mit persönlicher Schutzausrüstung dar [10, 18, 19, 22].

\section{Strukturelle Maßnahmen}

Insgesamt 29 Publikationen berichten von organisationalen und personellen Umstrukturierungsmaßnahmen als Reaktion auf die COVID-19-Pandemie. Zu ersteren gehört die Einteilung der Einrichtung in verschiedene Zonen, die Patienten mit Verdacht auf oder bestätigter COVID19-Infektion von Patienten trennt, die negativ auf COVID-19 getestet wurden [15, 23-25]. Zu den personellen Umstrukturierungsmaßnahmen gehören die Bildung von festen Mitarbeiterkohorten und die Zuordnung dieser zu festen Patienten, um ggf. auftretende Infektionen auf eine kleinere Gruppe von Kontaktpersonen zu beschränken [15, 19, 26].

Um Personalengpässen entgegenzuwirken, kann auf Zeitarbeitspersonal zurückgegriffen, das Personal innerhalb der Einrichtung umverteilt oder Urlaubssperren verhängt werden [13]. Andere Artikel berichten darüber hinaus von der Rekrutierung von Personal aus der arbeitslosen Bevölkerung [18] oder aus einem Pool von Medizinstudierenden [27]. Strategien zur Bewältigung knapper materieller Ressourcen stellen die kontrollierte Ausgabe und Rationierung von Schutzausrüstung sowie die eigene Herstellung von Schutzmaterialien und Desinfektionsmitteln dar [13]. Zudem können Träger, Verbände, Ämter und Behörden sowie Angehörige bei der Beschaffung von Schutzmaterialien zur Hilfe gezogen werden [13].

\section{Besuchsregelungen}

Zahlreiche Einrichtungen $(n=6)$ berichten von einer Änderung der Besuchsregelungen im Rahmen der COVID-19-Pandemie, die sich sowohl in der Beschränkung der Zahl von Besuchern [22, 28-30] wie auch der Verkürzung von Besuchszeiten widerspiegelt $[29,31]$. Einige Publikationen $(n=3)$ empfehlen die Einführung von Ausnahmeregelungen für Patienten am Lebensende $[4,21,26]$. Es kann beispielsweise ein gemeinsamer oder gestaffelter Besuch mehrerer Angehöriger in einem Einzelzimmer mit guter Lüftungsmöglichkeit ermöglicht werden. Falls ein einrichtungsinterner Besuch aufgrund hoher Infektionszahlen nicht möglich ist, sollten Möglichkeiten im Freien geschaffen werden, wie beispielsweise Balkon-/ Fensterbesuche oder Besuche über die Terrasse bzw. den Garten [26]. Besucher sollten während der Besuche einlagige Kleidung tragen, Schmuck ablegen und Wertsachen begrenzen [32].

\section{Kommunikationsstrukturen}

Um einen physischen Kontakt zu vermeiden, aber dennoch soziale Interaktionen zu ermöglichen, wurde vielfach der Einsatz digitaler Kommunikationstools thematisiert $(n=28)[4,9,12,13,17,19,21$, 22, 24, 26-28, 31, 33-48]. Videokommunikationstools wie Zoom oder Skype können sowohl für die Kommunikation zwischen Personal und Patienten als auch für den Kontakt zwischen Patienten und ihren Angehörigen nützlich sein [4, 21, 43]. Kliniken sollten für die Nutzung solcher Videokommunikationstools Smartphones, Tablets und Laptops sowie einen kostenlosen Internetzugang bereitstellen [21, 33, 34, 37-39]. Eine weitere Möglichkeit des virtuellen Dienstes stellt die virtuelle Realität (VR) dar, die jedoch mit hohen Kosten verbunden ist [43].

Um Hindernissen bei der Kommunikation mittels Mund-Nasen-Bedeckungen entgegenzuwirken, empfehlen einige Artikel $(n=3)$ Mitarbeitern und Besuchern deutlicher, langsamer und/oder lauter zu sprechen, verstärkte gestische Kommunikation anzuwenden [19] oder auch durchsichtige Gesichtsmasken zu verwenden [33, 49].

\section{Schulung und Aufklärung}

Um die Eigenverantwortlichkeit und das Gefahrenbewusstsein der Mitarbeiter zu fördern, führten einige Einrichtungen Schulungen zur praktischen Umsetzung der Hygienemaßnahmen, zum korrekten An- und Ablegen der persönlichen Schutzausrüstung und zum Umgang mit digitalen Kommunikationsmitteln durch $[12,23,37,50]$. Neben der Schulung des Personals sollten auch Patienten und ihre Angehörigen über die aktuelle Lage der COVID-19-Pandemie und die geltenden Richtlinien aufgeklärt werden [26]. Für die Schulung können beispielsweise E-Learn-
ing-Plattformen [13], Hands-on-Trainings [19], Aushänge in der Einrichtung [26] oder auf die jeweilige Zielgruppe zugeschnittene Aufklärungsmaterialien, z. B. in Form von Flyern oder Videoclips [26, 50], eingesetzt werden.

\section{Psychosoziale Unterstützungs- maßnahmen}

Um einer psychischen und physischen Überlastung des Personals entgegenzuwirken, wird vorgeschlagen, diesen Rückzugsräume in der Einrichtung, genügend Pausen und eine gute Verpflegung bereitzustellen [4]. Die Leitungspersonen der Gesundheitseinrichtungen sollten eine vertrauensvolle Atmosphäre schaffen [19], Kommunikationsbereitschaft signalisieren und Mitarbeiter in Entscheidungen einbeziehen [13, 51]. Um die Motivation der Mitarbeiter zu erhöhen, stellten einige Einrichtungen zusätzliche Getränke, Gutscheine, Verpflegungsgeld, kostenlose Parkplätze, Supervisionen, Kinderbetreuungshilfen und virtuelles Yoga zur Verfügung [13, 24].

Um einer sozialen Isolation und Vereinsamung der Patienten entgegenzuwirken, können diesen aufgezeichnete Hörbücher, Karten/Briefe, gemalte Bilder oder Fotos der Angehörigen zur Verfügung gestellt werden [4, 35, 52, 53]. Weiterhin können die Versorgungsanbieter Konzerte, Gottesdienste oder Geburtstage im Außenbereich der Einrichtung veranstalten, an denen die Patienten vom Fenster oder vom Balkon aus teilnehmen können [19, 26]. Sowohl Patienten und ihren Angehörigen als auch Mitarbeitern sollte ein Zugang zu einrichtungsinternen und -externen Fachkräften, die eine psychosoziale Betreuung anbieten, ermöglicht werden $[4,19,34$, 54].

\section{Spezifische Überlegungen für Menschen mit Demenz}

Demenzerkrankte Patienten sollten gesondert bei der ordnungsgemäßen Anwendung der Hygienemaßnahmen unterstützt werden. Dazu können beispielsweise demenzfreundliche Hinweistafeln, die an die Händehygiene erinnern, angebracht werden. Um die Einhaltung physischer Distanzierungsmaßnahmen bei Menschen 
mit Demenz sicherzustellen, können Indoor-Hobbys nützlich sein. Zur Vorbeugung von Ängsten im Zusammenhang mit der COVID-19-Pandemie sollten negative Ausdrücke wie „Krise", "Pandemie" oder "Isolierung" in Gegenwart der Demenzerkrankten vermieden werden. Auch bei der Nutzung digitaler Kommunikationstools benötigen Menschen mit Demenz zusätzliche Unterstützung durch die Mitarbeiter [25].

\section{Vorausschauende Pflegeplanung}

Versorgungsanbieter sollten rechtzeitig Gespräche zur Vorausplanung der Pflege mit den Patienten und ihren Angehörigen vereinbaren, um über Therapieziele, Behandlungspräferenzen, den bevorzugten Behandlungs-/Sterbeort [17, 19, 25, 26, 34, 54], gewünschte Rituale und Bestattungs-/ Gedenkpläne zu sprechen [55] und unerwünschte und belastende Behandlungen zu vermeiden [25, 54]. In Leitlinien wird zudem empfohlen, bei vorhandenen $\mathrm{Pa}$ tientenverfügungen aus der Zeit vor der COVID-19-Pandemie die aktuelle Relevanz zu überprüfen [19, 26, 54].

\section{Abschiednahme}

Mehreren Angehörigen sollte, entweder gemeinsam oder zeitlich versetzt, eine Abschiednahme im nahen Umfeld des Patienten unter Einhaltung grundlegender Hygienemaßnahmen gestattet werden $[25,26]$. Sofern dies nicht möglich ist, sollten digitale Kommunikationsmittel [25] oder auch Fotos der Verstorbenen zum Abschiednehmen genutzt werden [21, 52, 56]. Falls es die Infektionslage zulässt, sei es zudem empfehlenswert, Handabdrücke oder ein Herzbild der sterbenden Person anzufertigen. Diese können die Hinterbliebenen nach dem Tod mit den Patienten verbinden [36]. Die Deutsche Gesellschaft für Palliativmedizin empfiehlt darüber hinaus zur Erleichterung des Prozesses der Abschiednahme für die Angehörigen den Rückgriff auf Palliativpsychologen als Vermittler zwischen Klinik und Angehörigen [52].

\section{Maßnahmen nach dem Tod}

Einrichtungen sollten Hinterbliebene proaktiv auf Unterstützungsdienste zur Trauerbewältigung hinweisen [26], ihnen aktuelle Informationen für die Organisation einer Beerdigung bereitstellen und Familien in sozioökonomischen Risikosituationen einen Hinweis auf öffentliche Beihilfen geben [34, 57]. Falls die Teilnahme an der Bestattung aufgrund der Pandemie nur wenigen Personen gewährleistet werden kann, sollte auf digitale Möglichkeiten verwiesen werden [26].

\section{Diskussion}

Die eingeschlossenen Publikationen zeigen auf, dass bei der Bewältigung der Pandemie v.a.Kommunikationsstrategien, Empfehlungen zur psychosozialen Unterstützung aller Akteure, strukturelle Strategien und Schulungs- und Aufklärungsmaßnahmen dominieren. Es ist jedoch festzuhalten, dass bei Personalengpässen eine Aufteilung in kleine Mitarbeiterteams und eine Zuordnung dieser auf einzelne Patienten organisatorisch schwer umsetzbar ist. Auch die Bildung von Isolierstationen kann aufgrund von fehlenden räumlichen Kapazitäten erschwert sein [58]. Die in fast allen Publikationen thematisierte Nutzung digitaler Kommunikationstools kann für körperlich und kognitiv eingeschränkte Patienten schwierig sein. Zudem ist die Anschaffung notwendiger IT-Technik mit erhöhten Kosten verbunden, welche die Einrichtungen im Rahmen der derzeitigen Pandemie möglicherweise nicht abdecken können und daher entsprechend finanziell und strukturell unterstützt werden müssen [59].

Nur eine Minderheit der eingeschlossenen Artikel gibt gesonderte Empfehlungen für die Palliativ- und Hospizversorgung von Menschen mit Demenz in Pandemiezeiten aus. Dies stellt ein Beispiel für eine vulnerable Bevölkerungsgruppe dar, der vergleichsweise wenig Aufmerksamkeit gewidmet wurde [25]. Es bedarf demnach bei der Entwicklung von Strategien und Empfehlungen zur Bewältigung von Public-Health-Krisen einer erhöhten Aufmerksamkeit für besonders vulnerable Zielgruppen. Weiterhin gibt es nur eine geringe Anzahl an Publikationen, die sich mit der Abschiednahme und Maßnahmen nach dem Tod, wie z. B. der Trauerbewältigung von Hinterbliebenen oder Bestattungsvorgängen in Pandemiezeiten, beschäftigen [60]. Nur ein geringer Anteil der eingeschlossenen Artikel gibt Empfehlungen zur vorausschauenden Pflegeplanung aus. Gespräche zur Vorausplanung der Pflege sind insbesondere in Zeiten von COVID-19 unerlässlich, da sie unerwünschte lebenserhaltende Behandlungen verhindern und damit auch Ressourcen des Gesundheitssystems, die in Pandemiezeiten umso beschränkter sind, schonen [61].

\section{Fazit für die Praxis}

- Es besteht ein Bedarf an Studien zu: Palliativversorgung von Menschen mit Demenz in Zeiten von COVID-19, Abschiednahme und Maßnahmen nach dem Tod.

- Experten aus dem Bereich der Palliativund Hospizversorgung, Patienten und ihre Angehörigen sollten in die Entwicklung von Leitlinien einbezogen werden.

- Identifizierte Strategien und Empfehlungen sollten in der Praxis erprobt und evaluiert werden.

\section{Korrespondenzadresse}

Prof. Dr. PH Patrick Brzoska, MSc, EMPH

Fakultät für Gesundheit/Department für Humanmedizin, Lehrstuhl für Versorgungsforschung, Universität Witten/Herdecke Alfred-Herrhausen-Str. 50, 58448 Witten, Deutschland

patrick.brzoska@uni-wh.de

Förderung. Bundesministerium für Bildung und Forschung (Förderkennzeichen: 01 KI20126).

Funding. Open Access funding enabled and organized by Projekt DEAL.

\section{Einhaltung ethischer Richtlinien}

Interessenkonflikt. D. Wahidie, K. Altinok, Y. YılmazAslan und P. Brzoska geben an, dass kein Interessenkonflikt besteht.

Für diesen Beitrag wurden von den Autoren keine Studien an Menschen oder Tieren durchgeführt. Für die aufgeführten Studien gelten die jeweils dort angegebenen ethischen Richtlinien.

Open Access. Dieser Artikel wird unter der Creative Commons Namensnennung 4.0 International Lizenz veröffentlicht, welche die Nutzung, Vervielfältigung, Bearbeitung, Verbreitung und Wiedergabe in jeglichem Medium und Format erlaubt, sofern Sie den/die 
ursprünglichen Autor(en) und die Quelle ordnungsgemäß nennen, einen Link zur Creative Commons Lizenz beifügen und angeben, ob Änderungen vorgenommen wurden.

Die in diesem Artikel enthaltenen Bilder und sonstiges Drittmaterial unterliegen ebenfalls der genannten Creative Commons Lizenz, sofern sich aus der Abbildungslegende nichts anderes ergibt. Sofern das betreffende Material nicht unter der genannten Creative Commons Lizenz steht und die betreffende Handlung nicht nach gesetzlichen Vorschriften erlaubt ist, ist für die oben aufgeführten Weiterverwendungen des $\mathrm{Ma}$ terials die Einwilligung des jeweiligen Rechteinhabers einzuholen.

Weitere Details zur Lizenz entnehmen Sie bitte der Lizenzinformation auf http://creativecommons.org/ licenses/by/4.0/deed.de.

\section{Literatur}

1. OngEK,Soh GT,PehTYetal (2020)Challengesfaced by community palliative care services during the COVID-19 pandemic-experiences from a hospice. Ann Acad Med Singap 49:588-593

2. Jordan RE, Adab P, Cheng KK (2020) Covid-19: risk factors for severe disease and death. BMJ 368:m1198

3. Schweickert B, Klingeberg A, Haller S et al (2021) COVID-19-Ausbrüche in deutschen Alten- und Pflegeheimen. Epid Bull 18:3-29

4. Münch U, Müller H, Deffner T et al (2020) Empfehlungen zur Unterstützung von belasteten, schwerstkranken, sterbenden und trauernden Menschen in der Corona-Pandemie aus palliativmedizinischer Perspektive. Schmerz 34:303-313

5. Arksey H, O'Malley L (2005) Scoping studies: towards a methodological framework. Int J Soc Res Methodol 8:19-32

6. Levac D, Colquhoun H, O'Brien KK (2010) Scoping studies: advancing the methodology. Implement Sci 5:69

7. Peters MDJ, Godfrey CM, Khalil $\mathrm{H}$ et al (2015) Guidance for conducting systematic scoping reviews. Int JEvid Based Healthc 13:141-146

8. Tricco AC, Lillie E, Zarin W et al (2018) PRISMA extension for scoping reviews (PRISMA-scR): checklist and explanation. Ann Intern Med 169:467-473

9. Apoeso O, Kuwata C, Goldhirsch SL et al (2021) Creating a colocation unit for end-of-life care during a pandemic. J Palliat Med 24:574-579

10. Mitchell S, Maynard V, Lyons V et al (2020) The role and response of primary healthcare services in the delivery of palliative care in epidemics and pandemics: a rapid review to inform practice and service delivery during the COVID-19 pandemic. PalliatMed 34:1182-1192

11. Boufkhed S, Harding R, Kutluk T et al (2021) What is the preparedness and capacity of palliative care services in middle-eastern and north African countries to respond to COVID-19? A rapid survey. J Pain Symptom Manage 61:e13-e50

12. Boufkhed S, Namisango E, Luyirika E et al (2020) Preparedness of African palliative care services to respond to the COVID-19 pandemic: a rapid assessment.J Pain Symptom Manage 60:e10-e26

13. Hower Kl, Pfaff H, Pförtner T-K (2020) Pflege in Zeiten von COVID-19: Onlinebefragung von Leitungskräften zu Herausforderungen, Belastungen und Bewältigungsstrategien. Pflege 33:207-218
14. Powell VD, Silveira MJ (2020) What should palliative care's response be to the COVID-19 pandemic? JPain Symptom Manage 60:e1-e3

15. Kluge S, Janssens U, Welte T et al (2021) S3Leitlinie -Empfehlungen zur stationären Therapie von Patienten mit COVID-19. www.awmf.org/ leitlinien/detail/l//113-001LG.html. Zugegriffen: 14. Januar 2022

16. van Oorschot $B$, Haberland $B$, Simon $S$ et al (2020) Vorschläge für die Arbeitsweise von Palliativdiensten (PD) in der COVID-19-Pandemie. https://www.dgpalliativmedizin.de/images/ 200321_DGP_Vorschl\%C3\%A4ge_f\%C3\%BCr die_Arbeitsweise_von_Palliativdiensten_in_der_ COVID-19-Pandemie.pdf. Zugegriffen: 14. Januar 2022

17. Delis S, Nehls W, Maier BO et al (2020) Handlungsempfehlung zur Sicherstellung der Palliativversorgung in Zeiten der COVID-19-Pandemie. https:// www.dgpalliativmedizin.de/images/200319_ DGP_Handlungsempfehlung_Sicherstellung_ Palliativversorgung_w\%C3\%A4hrend_COVID19-Pandemie.pdf.Zugegriffen: 14.Januar 2022

18. Cohen-Mansfield J (2020) COVID-19 and older adults in Israel-common challenges and recommendations. Qual Ageing Older Adults 21:209-216

19. Halek M, Holle D (2020) Soziale Teilhabe und Lebensqualität in der stationären Altenhilfe unter den Bedingungen der COVID-19-Pandemie. www.awmf.org/leitlinien/detail/ll/184-001.html. Zugegriffen: 14. Januar 2022

20. Lazzarin P, Avagnina I, Divisic A et al (2020) Management strategies adopted by a paediatric palliative care network in northern Italy during the COVID-19 pandemic. Acta Paediatr 109:1897-1898

21. Schwartz J, Reuters MC, Schallenburger $M$ et al (2021) General palliative care during the pandemic. Onkologe 27:686-690

22. Luckett T, Donkor A, Phillips Jet al (2021) Australian specialist palliative care's response to COVID-19: an anonymous online survey of service providers. Ann Palliat Med 10:2747-2757

23. Abdihamid O, Cai CJ, Kapesa L et al (2020) The landscape of COVID-19 in cancer patients: prevalence, impacts, and recommendations. Cancer Manag Res 12:8923-8933

24. Dunleavy L, Preston N, Bajwah S et al (2021) "Necessity is the mother of invention": specialist palliative care service innovation and practice change in response to COVID-19. Results from a multinational survey (Covpall). Palliat Med 35:814-829

25. Bolt SR, van der Steen JT, Mujezinović let al (2021) Practical nursing recommendations for palliative care for people with dementia living in long-term care facilities during the COVID-19 pandemic: a rapid scoping review. Int J Nurs Stud 113:103781

26. Bausewein C, Simon S (2021) Nationale Strategie für die Betreuung von schwerkranken und sterbenden Menschen und ihren Angehörigen in Pandemiezeiten (PallPan) https://doi.org/10. 5281/zenodo.5012504

27. Dhahri AA, Thabrew AU, Ladva N et al (2021) The benefits and risks of the provision of a hospitalwide high-definition video conferencing virtual visiting service for patients and their relatives. Cureus 13(2):e13435. https://doi.org/10.7759/ cureus. 13435

28. Kates J, Gerolamo A, Pogorzelska-Maziarz M (2021) The impact of COVID-19 on the hospice and palliative care workforce. Public Health Nurs 38:459-463
29. Hsu Y-C, Liu Y-A, Lin M-H et al (2020) Visiting policies of hospice wards during the COVID-19 pandemic: an environmental scan in Taiwan. Int J Environ Res Public Health 17(8):2857. https://doi. org/10.3390/ijerph17082857

30. Costantini M, Sleeman KE, Peruselli C et al (2020) Response and role of palliative care during the COVID-19 pandemic: a national telephone survey of hospices in Italy. Palliat Med 34:889-895

31. Etkind SN, Bone AE, Lovell N et al (2020) The role and response of palliative care and hospiceservices in epidemics and pandemics: a rapid review to inform practice during the COVID-19 pandemic J Pain Symptom Manage 60:e31-e40

32. Bloomer MJ, Bouchoucha S (2021) Australian college of critical care nurses and Australasian college for infection prevention and control position statement on facilitating next-of-kin presence for patients dying from coronavirus disease 2019 (COVID-19) in the intensive care unit. Aust Crit Care 34:132-134

33. Ong KJ, Lim MYY, Chng JXR et al (2020) Collateral damage: how the COVID-19 pandemichas affected the dying process of palliative care patients in hospitals-our experience and recommendations. Ann Acad Med Singap 49:616-620

34. Selman LE, Chao D, Sowden R et al (2020) Bereavement support on the frontline of COVID19: recommendations for hospital clinicians. J Pain Symptom Manage 60:e81-e86

35. Pearce C, Honey JR, Lovick R et al (2021) „A silent epidemic of grief": a survey of bereavement care provision in the UK and Ireland during the COVID19 pandemic. BMJ Open 11:e46872

36. Morris SE, Moment A, deLima Thomas J (2020) Caring for bereaved family members during the COVID-19 pandemic: before and after the death of a patient. J Pain Symptom Manage 60:e70-e74

37. Maaskant JM, Jongerden IP, Bik J et al (2021) Strict isolation requires a different approach to the family of hospitalised patients with COVID-19: a rapid qualitative study. Int J Nurs Stud 117:103858

38. Hart JL, Turnbull AE, Oppenheim IM et al (2020) Family-centered care during the COVID-19 era. JPain Symptom Manage 60:E93-E7

39. Humphreys J, Schoenherr L, Elia Getal (2020) Rapid implementation of inpatient telepalliative medicine consultations during COVID-19 pandemic. JPain Symptom Manage 60:e54-e59

40. Florencio RS, Cestari VR, Souza LC et al (2020) Palliative care amidst the COVID-19 pandemic: challenges and contributions. Acta paulista de enfermagem. https://doi.org/10.37689/acta-ape/ 2020a001886

41. Davies A, Hayes J (2020) Palliative care in the context of a pandemic: similar but different. Clin Med 20(3):274-277. https://doi.org/10.7861/ clinmed.2020-0157

42. Flores S, Abrukin L, Jiang L et al (2020) Novel use of telepalliative care in a New York City emergency department during the COVID-19 pandemic. JEmerg Med 59:714-716

43. Cherniwchan HR (2021) Harnessing new and existing virtual platforms to meet the demand for increased inpatient palliative care services during the COVID-19 pandemic: a 5 key themes literature review of the characteristics and barriers of these evolving technologies. Am J Hosp Palliat Care. https://doi.org/10.1177/10499091211036698

44. Blinderman $C D$, Adelman $R$, Kumaraiah $D$ et al (2021) A comprehensive approach to palliative care during the coronavirus pandemic. J Palliat Med 24:1017-1022 
45. Pickell Z, Gu K, Williams AM (2020) Virtual volunteers: the importance of restructuring medical volunteering during the COVID-19 pandemic. Med Humanit 46:537-540

46. Reinhard MA, Burkhardt G, Grosse-Wentrup F et al (2021) Psychosoziale Unterstützung während der COVID-19-Pandemie: interdisziplinäres Versorgungskonzept an einem Universitätsklinikum. Nervenarzt 92:701-707

47. Haire E, Brown H, Wiggins N (2021) „Compassion outside of the box": the role of allied healthcare professionals in providing a companion service for patients at the end of life during the COVID-19 pandemic. J Pain Symptom Manage 62:141-148.e2

48. Adams C (2020) Goals of care in a pandemic: our experience and recommendations. J Pain Symptom Manage 60:e15-e17

49. Gergerich E, Mallonee J, Gherardi S et al (2021) Strengths and struggles for families involved in hospice care during the COVID-19 pandemic. J Soc WorkEnd Life PalliatCare. https://doi.org/10.1080/ 15524256.2020.1845907

50. Griffin KM, Karas MG, Ivascu NS et al (2020) Hospital preparedness for COVID-19: a practical guide from a critical care perspective. Am J Respir Crit Care Med 201:1337-1344

51. Hofmeyer A, Taylor R (2021) Strategies and resources for nurse leaders to use to lead with empathy and prudence so they understand and address sources of anxiety among nurses practising in the era of COVID-19. J Clin Nurs 30:298-305

52. Berthold D, Gramm J, Hofmann S et al (2020) COVID-19-Pandemie. Impulse für Palliativpsycholog*innen. https://www.dgpalliativmedizin. de/images/COVID_19_Sektion_Psychologie.pdf. Zugegriffen: 14. Januar 2022

53. Chomton M, Marsac L, Deho A et al (2021) Transforming a paediatric ICU to an adult ICU for severe Covid-19: lessons learned. Eur J Pediatr 180:2319-2323

54. Janssen DJA, Ekström M, Currow DC et al (2020) COVID-19: guidance on palliative care from a European respiratory society international task force. Eur Respir J 56(3):2002583

55. Wallace $\mathrm{CL}$, Wladkowski SP, Gibson A et al (2020) Grief during the COVID-19 pandemic: considerations for palliative care providers. J Pain Symptom Manage 60:E70-E76

56. Roser T, Peng-Keller S, Kammerer T et al (2020) Die CoronaPandemieals Herausforderung für Spiritual Care: Hinweise für Seelsorger*innen. https://www. uni-muenster.de/imperia/md/content/evtheol/ personen/2020_03_25_die_corona-pandemie als_herausforderung_f_r_spiritual_care.pdf. Zugegriffen: 14. Januar 2022

57. Beneria A, Castell-Panisello E, Sorribes-Puertas M et al (2021) End of life intervention program during COVID-19 in Vall d'Hebron university hospital. Front Psychiatry 12:608973. https://doi.org/10. 3389/fpsyt.2021.608973

58. Bundesministerium für Soziales, Gesundheit, Pflege und Konsumentenschutz (BMSGPK) (2020) COVID-19 in Alten- und Pflegeheimen. https:// goeg.at/COVID-19_APH. Zugegriffen: 14. Januar 2022

59. Dobbs D, Peterson L, Hyer K (2020) The unique challenges faced by assisted living communities to meet federal guidelines for COVID-19. J Aging Soc Policy 32:334-342

60. Gilissen J, Pivodic L, Unroe KT et al (2020) International COVID-19 palliative care guidance for
Strategies, guidelines and recommendations for coping with the COVID-19 pandemic in palliative and hospice care facilities. Results of a scoping review

Background: Palliative and hospice care facilities face different challenges emerging from the COVID-19 pandemic. In particular, this results from the high age and preexisting diseases of patients as well as the physically close contact between staff and patients.

Objective: The aim of this study was to identify existing strategies, guidelines and recommendations in Germany and other countries with respect to addressing the challenges of the COVID-19 pandemic in palliative and hospice care.

Material and methods: The databases PubMed, CINAHL, Web of Science and PsycInfo were searched as part of a scoping review. Additionally, Google Search and Google Scholar were used to identify "grey literature". All German and English language articles related to inpatient palliative and hospice care were included which were published between January 2020 and August 2021.

Results: A total of 51 publications were included in the analysis. The identified measures can be clustered into 10 different categories: infection control measures, structural measures, visiting regulations, communication structures, education and training, psychosocial support measures, specific considerations for people with dementia, advance care planning, farewell and measures after death.

Conclusion: With respect to addressing the COVID-19 pandemic, the primary focus is on measures to enable communication between patients, staff and relatives, psychosocial support measures, structural measures and recommendations for training and education of all stakeholders. Measures for the palliative care of people with dementia in times of a pandemic as well as the farewell and grief management of bereaved families received less attention.

\section{Keywords}

SARS-CoV-2 - Measures · Social distancing · Infection control · Public health crisis

nursing homes leaves key themes unaddressed.

JPain Symptom Manage 60:e56-e69

61. Curtis JR, Kross EK, Stapleton RD (2020) The importance of addressing advance care planning and decisions about do-not-resuscitate orders during novel Coronavirus 2019 (COVID-19). JAMA 323:1771-1772

62. Bains J, Greenwald PW, Mulcare MR et al (2021) Utilizing telemedicine in a novel approach to COVID-19 management and patient experience in the emergency department. Telemed J E Health 27:254-260 